\title{
OVERALL MASS TRANSFER RATES FROM FRONT PORTIONS AND WAKE AREAS OF SPHEROIDS
}

\author{
SHAFKAT A. BEG \\ School of Engineering, Pahlavi University, Shiraz, Iran
}

\section{Introduction}

The study of mass transfer between two phases when one is dispersed into the other, is of considerable importance to many unit operations of Chemical Engineering as for example: absorption, extraction, distillation, drying and humidification. In order to obtain an insight into the mechanism of the process, it is customary to study transfer from single drops. Most of the research work devoted to mass transfer to or from the drops has been confined to studies in which a spherical shape has been assumed as a model for obtaining theoretical and empirical relations. But, from the photographic evidence, it appears than in many situations drops are non-spherical. Frequently, oblate spheroidal and shapes distorted more at the front half than the rear have been observed ${ }^{4-7,14-17,19-24,26,28)}$.

In the presence of surface-active agents, or for particular combination of physical properties, drops in the dispersed phase may behave like solid bodies being internally stagnant and non-oscillating ${ }^{3,8,9,18,29)}$. Empirical rate relationships obtained for rigid drop shapes would contribute towards the design of extraction columns.

Skelland and Cornish ${ }^{27)}$ used solid oblate spheroids of eccentricities (ratios of minor to major axes) (1:1) to $(1: 3)$ as a first stage to obtain an empirical expression for the prediction of continuous phase mass transfer coefficients. For limited range of Reynolds numbers $(120<\operatorname{Re}<6000)$ that they studied, they found that shape is an important factor which influences the transfer rates. In order to further describe and comprehend the transfer process from drops, it followed that other idealised drop shapes should also be considered as a basis for obtaining theoretical and experimental expressions for predicting transfer coefficients. Beg ${ }^{1)}$ studies overall forced convective mass transfer rates from oblate spheroids of eccentricities $(1: 1)$ to $(1: 4)$ and spheroids with composite halves having different eccentricities. The data obtained covered a large range of Reynolds numbers $270<\operatorname{Re}<34900$. It was concluded that

\footnotetext{
Received January 5, 1976.
}

shape is an important factor which influences the mass transfer rates. The application of relationships that have been presented for predicting continuous phase transfer coefficients for spheres, may lead to considerable error if applied to drops of other shapes. Therefore, in order to further explore the effect of shape, data were obtained for overall mass transfer rates from front portions up to the flow separation point and wake areas of spheroids for range of eccentricities.

\section{Experimental}

The mass transfer rates from spheroids were measured from local rates of sublimation of naphthalene spheroids rigidly mounted in the test section of a wind tunnel. An open circuit wind tunnel designed to produce an air stream of uniform velocity and low intensity of turbulence was used for the experiments. The flared inlet ensured a steady air stream and avoided separation of flow from the walls. In order to reduce turbulence, the inlet section was fitted with a honey comb flow straightener and a forty mesh screen. A contraction section that had an area ratio of $3: 1$ enabled the velocity distribution to be uniform at the test section. The test section was octagonal in cross-section, had a distance of 12 in. between opposite faces, was two and a half feet long and was fitted with perspex glass windows for observation and photographic purposes.

The diffuser was an expanding section with an outlet of the same diameter as the fan housing. The diffuser was separated from the fan shell by a thin rubber sheet which prevented the mechanical vibrations produced by the fan from being transmitted to the test section. As an additional safe guard the entire wind tunnel was mounetd on vibration reducing pads. The fan was an Airscrew company and Jigwood Ltd., standard 24.0 in. diameter unit. The fan was driven by Vee belts from the outlet shaft of Carter F-14 variable speed drive. The input shaft of variable speed drive was driven by a Brook 4-horse power electric motor.

\section{Production of spheroids}

There are various methods by which spheroids 
may be produced. One method is based on the application of thin coatings of volatile materials to the surface of solid metals. Coatings that are sprayed on, brushed on or obtained by dipping the models into the molten materials are usually deposited unevenly. Liquid coatings, even if extremely thin, are moved along the model surface by the air stream. The method ${ }^{10-13)}$ based on compression of materials in moulds fitted in a hand press have been found to suffer obvious disadvantages, the major one being that there is no accurate and simple way of fixing the support to the pelleted particles. For non-spherical shapes such as spheroids, accurate positioning of the support becomes even more difficult. Moreover, the compression method requires repeated applications of pressure in order to build up the layer upon the layer. It is quite possible that the stratified structure may cause irregular transfer and significantly affect the local measurements. Moreover, it would be difficult to produce spheroids by this technique. From these considerations, it was decided to cast the spheroids, since this technique ensures good accuracy and reproducibility. Further more there is no difficulty in fixing the support to the cast model.

Spheroids of eccentricities (i.e. ratios of minor to major axes) ranging from $16: 16$ to $4: 16$, and major axes of one inch were manufactured in specially designed stainless steel moulds ${ }^{1)}$. The shapes used were selected to provide a range of surface areas spaced at approximately equal intervals. The five sets employed were having eccentricities $16: 16,13: 16,10: 16,7: 16$ and $4: 16$.

Measurements of overall mass transfer rates from front portions and wake areas of spheroids

The overall mass transfer rates from front portions up to the separation point and wake areas of spheroids were measured from the local mass transfer distributions around spheroids. A double exposure photographic technique which eliminates the need for the exact location of two separate silhouettes of spheroidal model was devised for the local measurements of mass transfer rates ${ }^{2)}$. Two exposures were made, one before and one after the experimental run on the same photographic plate while the camera remained in an undisturbed position. The spheroids were rigidly mounted along the axis of the wind tunnel so as to ensure the fixed position of the spheroids. The exposures were made with the transmitted light and high resolving lens of the camera presented with silhouettes of nonilluminated spheroids against a bright back ground. The intensities of the light for the two exposures were so adjusted that the photographic negative consisted of three regions, the outer dark boundary

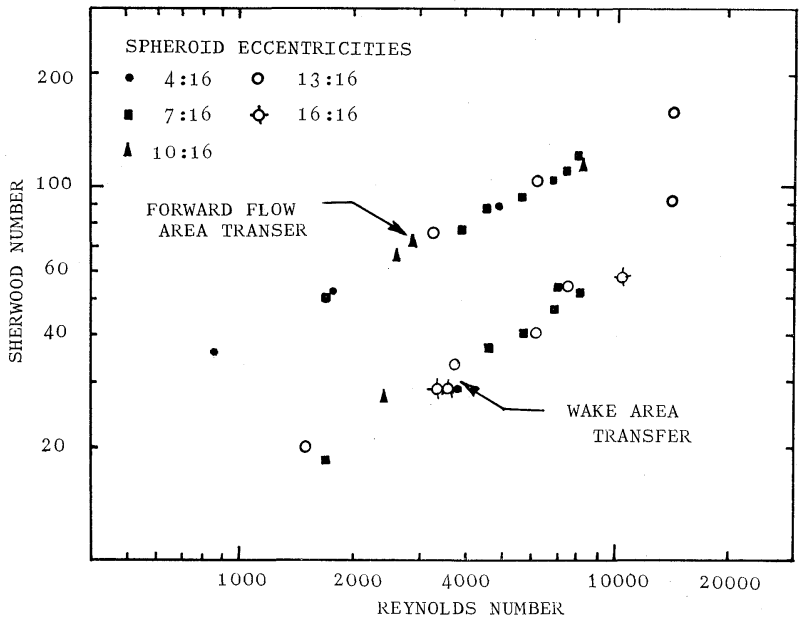

Fig. 1 Data for mass transfer from forward flow and wake areas of spheroids

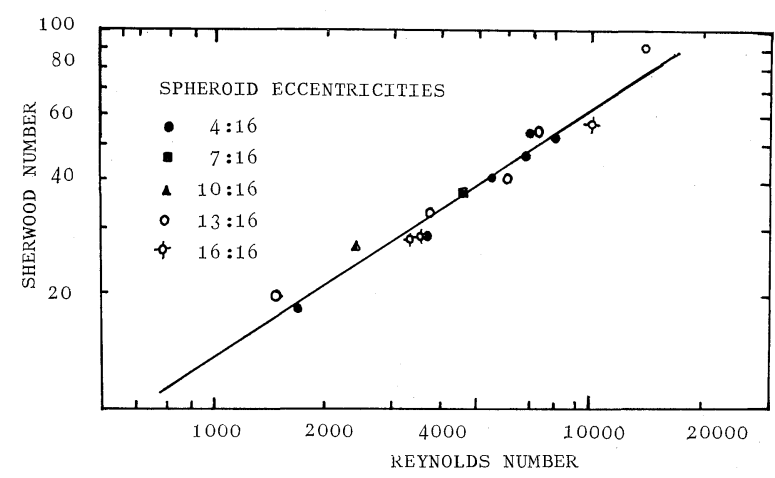

Fig. 2 Data for mass transfer from wake areas of spheroids

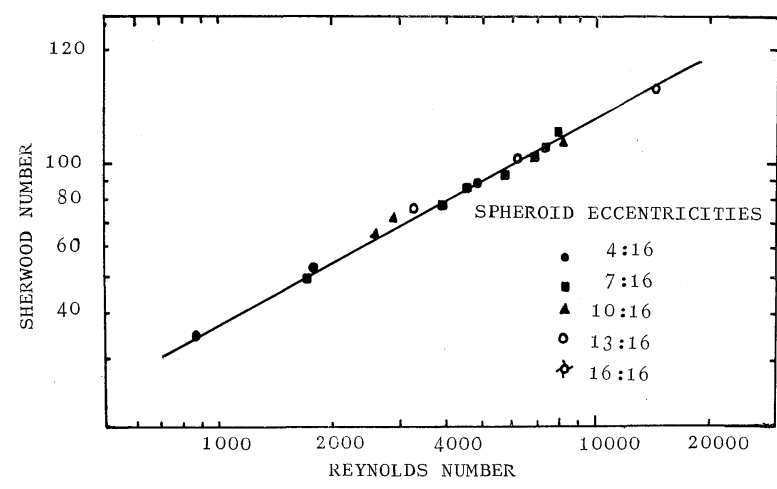

Fig. 3 Data for mass transfer from front portions of spheroids

representing the original size of the spheroids, the light shaded portion representing the diminution, and the transparent region the final size. Measurements of the dimensional changes were made from images of the spheroids enlarged to twenty times the original size. The definition of the enlarged images was very good, and no difficulty was experienced in measuring the diminutions with an accuracy that corresponded to an actual dimensional change of $0.01 \mathrm{~mm}$. Local mass transfer rates were measured from the diminutions measured at succes- 
sive intervals of ten degrees around the peripheries of spheroidal shapes. The total amounts of mass transfer were calculated and compared with the amounts determined from direct weighings. The maximum and mean differences between the calculated and weighed amounts transferred were $5.8 \%$ and $3 \%$ of the weighed amount, respectively.

It is interesting to note that the point of separation which appeared on double exposure photographs as point of minimum mass transfer was found to be extremely close to $\left(\alpha=90^{\circ}\right)$ for spheroids of eccentricities of $4: 16,7: 16$ and $10: 16$, for the range of Reynolds number considered, where $(\alpha)$ is the oblate spheroidal co-ordinate measured from the front stagnation point. For spheroids of eccentricities $13: 16$ and $16: 16$, the separation point deviated to a maximum of $\left(\alpha=3^{\circ}\right)$ to that observed for other shapes. This was not considered to be a significant shift of separation point compared to other shapes and mass transfer data were analysed for front portions and wake areas of the spheroids based on a separation point of $\left(\alpha=90^{\circ}\right)$.

\section{Results and Discussion}

Fig. 1 shows the data for overall mass transfer rates from front portions and wake areas of spheroids plotted on logarithmic co-ordinate as Sherwood number against Reynolds number. Although various characteristic dimensions could be employed for the calculation of Sherwood number and Reynolds number, the characteristic dimension defined by Pasternak and Gauvin ${ }^{25)}$ as total surface area divided by maximum perimeter normal to flow was used. This characteristic dimension has been found to correlate the data for different shapes available in literature. The data were correlated by a relation of following form:

$$
S h=A+B(R e)(S c)^{1 / 3}
$$

where $A$ is an intercept value resulting from a linear data fitting. The least squares correlations were obtained by the minimization of the sum of squares of relative errors defined by following expression

$$
\sum_{t=1}^{t=q} R_{t}^{2}=\sum_{t=1}^{t=q}\left[\left(\overline{S h_{t}}-S h_{t}\right) / S h_{t}\right]^{2}
$$

where $q$ is the number of data points, $S h_{t}$ is the experimental value of the Sherwood number and $\overline{S h}_{t}$ is the predicted value of the Sherwood number for a particular data point $t$. It was found necessary to study the wake transfer rates from spheroids, in order to make an attempt to show that the exponent (n) for the Reynolds number, for this portion is significantly different from the theoretically predicted value of one half obtained for the front portions of spheroids. This may justify the result that it is not valid to make the assumption that the exponent $(n)$ is one half for overall transfer rates from solid bodies at high Reynolds numbers ${ }^{1}$. The following correlations for the data for wake transfer from spheroids were obtained:

$$
\begin{aligned}
& S h=10.95+0.0041(R e)^{0.99}(S c)^{1 / 3} \\
& S^{2}(\bar{Y})=9.21 \times 10^{-3} \\
& S h=0.10+0.66(R e)^{0.50}(S c)^{1 / 3} \\
& S^{2}(\bar{Y})=1.12 \times 10^{-2} \\
& S h=0.12(R e)^{0.6}(S c)^{1 / 3} \\
& S^{2}(\bar{Y})=1.13 \times 10^{-2}
\end{aligned}
$$

In Eq. (4) the exponent ( $n$ ) was assumed to be 0.50 and in Eq. (5), $A$ was assumed to be zero, whereas in Eq. (3), the intercept $(A)$, coefficient $(B)$ and exponent $(n)$ were all estimated. Both the variances of the correlations given by Eqs. (4) and (5), were found to be within the $90 \%$ confidence range, obtained for the smallest variance $S^{2}(\bar{Y})=9.2 \times 10^{-3}$ of Eq. (3). It follows, therefore, that it is not invalid to assume a value of one-half for the exponent $(n)$ of the Reynolds number for the data for the wake region of spheroids. It may be because of the limited amount of data analysed that it is not possible to show that the exponent for the Reynolds number for the wake region of spheroids is significantly different from one-half.

The correlation give by Eq. (5) is plotted in Fig. 2 and fits the data with a standard deviation of $10.6 \%$. The data for overall transfer rates from the forward flow area of the spheroids was correlated and the following correlations were obtained.

$$
\begin{aligned}
& S h=-5.87+1.02(R e)^{0.5}(S c)^{1 / 3} \\
& S^{2}(\bar{Y})=1.56 \times 10^{-3} \\
& S h=0.67(R e)^{0.54}(S c)^{1 / 3} \\
& S^{2}(\bar{Y})=1.64 \times 10^{-3}
\end{aligned}
$$

In Eq. (6), the exponent of the Reynolds number (n) was assumed to be one-half, whereas, in Eq. (7) the intercept $(A)$ was assumed to be zero. The correlation given by Eq. (7) was preferred for its more convenient form and is plotted in Fig. 3. It was found, however, that the variance $S^{2}(\bar{Y})=1.64 \times$ $10^{-3}$ was included in the $90 \%$ confidence limits set on the variance associated with Eq. (6).

Literature survey shows that there is no other experimental or theoretical expression available for forced convective mass transfer from spheroids which could enable comparison with the present data.

\section{Conclusions}

Correlations have been obtained for transfer rates from frontal and wake areas of spheroids. It was 
not possible to conclude from the data obtained that the exponent ( $n$ ) of the Reynolds number was significantly different from one-half for the data for the wake regions of spheroids.

\section{Nomenclature}

$A \quad=$ constant in Eq. (1)

$B \quad=$ constant in Eq. (1)

$d \quad=$ characteristic dimension of spheroid

$D_{v} \quad=$ diffusion coefficient

$k_{c} \quad=$ continuous phase mass transfer coefficient

[cm]

[cm/sec]

$q \quad=$ number of observations

$R_{1}^{2}, \ldots \quad=$ error quantities defined by Eq. (2)

$R e \quad=u d / \nu$

$S^{2}(Y) \quad=$ estimate of variance

Sc $\quad=\nu / D_{v}$

$S h \quad=k_{c} d / D_{v}$

$t \quad=$ number of data points

$u \quad=$ velocity of air stream

$\alpha=$ oblate spheroidal co-ordinate $(\pi / 2-\eta)$

$\nu \quad=$ kinematic viscosity $\left[\mathrm{cm}^{2} / \mathrm{sec}\right]$

$\quad \quad=$ predicted values

\section{Literature Cited}

1) Beg, S. A.: Warme und Stoffubertragung, 8, 127 (1975).

2) Beg, S. A., N. Domtrowski and A. R. H. Cornish: Chem. Eng. Sci., 23, 1157 (1968).

3) Chu, J. C., C. C. Taylor and D. J. Levy: Ind. Eng. Chem., 42, 1157 (1950).

4) Egerton, H. E., E. A. Hanser, B. H. Hold and J. F. Cox: J. Phys. Chem., 40, 973 (1936).

5) Elzinga, E. R. and J. T. Banchero: AIChE J., 7, 394 (1961).

6) Garner, F. H.: Trans. Inst. Chem. Engrs., (London), 28,
95 (1950).

7) Garner, F. H., S. R. M. Ellis, and D. W. Fosbury: ibid., 31, 348 (1933).

8) Garner, F. H. and A. H. P. Skelland: Ind. Eng. Chem., 48, 51 (1956).

9) Garner, F. H. and A. R. Hale: Chem. Eng., 2, 157 (1953).

10) Garner, F. H. and J. M. Hoffman: AIChE J., 6, 579 (1960).

11) Garner, F. H. and J. M. Hoffman: ibid., 7, 148 (1961).

12) Garner, F. H. and R. D. Suckling: ibid., 4, 114 (1958).

13) Garner, F. H. and R. B. Keey: Chem. Eng. Sci., 9, 218 (1958).

14) Henrickson, F. Jr.: M. S. Thesis in Chemical Engineering, M.I.T. (1941).

15) Horton, T. J.: M. S. Thesis in Chemical Engineering Illinois Institute of Technology (1960).

16) Hu, S. and R. C. Kintner: AIChE J., 1, 42 (1955).

17) Hughes, R. R. and E. R. Gilliland: Chem. Eng. Prog., 48, 497 (1952).

18) Johnstone, H. F.: Trans. Am. Inst. Chem. Engrs., 35, 621 (1939).

19) Keith, F. W. Jr. and A. N. Hixson: Ind. Eng. Chem., 47, 258 (1955).

20) Klee, A. J. and R. E. Treybal: AIChE J., 2, 444 (1956).

21) Law, J. O.: Agric Eng., 21 (1940).

22) Lenard, P.: Uber Regen Meteor. Z., 21, 248 (1904).

23) Licht, W. and G. S. R. Narasimhamurty: AIChE J., 1, 366 (1955).

24) Magono, C.: J. Meteor., 11, 77 (1954).

25) Pasternak, I. S. and W. H. Gauvin: Can. J. of Chem. Eng., 38, 35 (1960).

26) Saito, S.: Sci. Rep. Tohoku Imp. Univ., 2, 179 (1913).

27) Skelland, A. H. P. and A. R. H. Cornish: AIChE J., 9, 73 (1963).

28) Spilhaus, A. E.: J. Meteor, 5, 108 (1948).

29) West, F., A. J., Harman, A. T. Chang and L. B. K. Thomas: Ind. Eng. Chem., 44, 625 (1952). 